\title{
Toward Patient-Friendly Cell-Level Dosimetry
}

D osimetry for targeted radionuclide therapy is a relatively young field. Although initial work can be traced back to the late 1940s (1), the MIRD Committee formalism that forms the foundation for most current approaches was published in the mid 1970s (2), and the drive for improved dosimetry to meet the requirements of targeted radionuclide therapy did not begin until the 1980s. During this period, the absorbed fraction and $\mathrm{S}$ value tables of MIRD Pamphlet 11 (3) and subsequently the values published by Cristy, Eckerman, and Stabin $(4,5)$ and implemented in the software package MIRDOSE3 (6) formed the basis for almost all dosimetry calculations.

\section{See page 645}

These data are traditionally characterized as making up the physics portion of the absorbed dose calculation; that is, they enable the conversion of the total number of radionuclide transformations in a particular source tissue to absorbed dose in a target tissue. Such a conversion requires information on the emission properties of the radionuclide as well as the sourcetarget tissue anatomy and composition. Given an appropriate model of human anatomy and composition, Monte Carlo calculations that track energy deposition events to different target regions from emissions in one or more source regions are used to generate photon or electron absorbed fractions for different

Received Nov. 3, 2006; revision accepted Nov. 9, 2006.

For correspondence or reprints contact: George Sgouros, PhD, CRB II 4M.61, 1550 Orleans St., Johns Hopkins University School of Medicine, Baltimore, MD 21231.

E-mail: gsgouros@jhmi.edu

COPYRIGHT (c) 2007 by the Society of Nuclear Medicine, Inc.

DOI: 10.2967/jnumed.106.036749 source-target combinations, and these data are then used to generate radionuclide $S$ values. These components are considered removed from the more difficult and messy biologic problem of determining the radiopharmaceutical kinetics needed to estimate the total number of radionuclide transformations in source tissues. Much of the effort in improving clinical radionuclide dosimetry over the past $10 \mathrm{y}$ has focused on the biologic part-establishing more accurate methods for collecting radiopharmaceutical kinetics, in vivo. These efforts have led to surrogate measures of pharmacokinetics $(7,8)$ and also to improved image quantitation methodologies tailored toward the radionuclides used in targeted radionuclide therapy (9-13).

Until recently, less attention had been paid to improving the physics input portion of the dose calculation method. Improvements in this area require more detailed anatomic models, both at the macroscopic level and at the microscopic level, and also improved Monte Carlo techniques. The need for such improvements has been greatest for the red bone marrow. The red bone marrow is the doselimiting organ in the majority of targeted radionuclide therapy. As has been previously reviewed $(14,15)$, the anatomic data used to generate the red marrow $\mathrm{S}$ values for the adult male of Pamphlet 11 and MIRDOSE3 came from a single 44-y-old man (16,17). Because the marrow mass was not available from this subject, the masses used to convert the absorbed fractions to $\mathrm{S}$ values came from studies dating to $1926(18,19)$. Also, because the absorbed fractions were calculated on the basis of measured chord-length distributions, the loss of energy to cortical bone from energetic $\beta$-emitters such as ${ }^{90} \mathrm{Y}$ could not be directly modeled, nor could be the reduction in marrow dose due to the variable fraction of hematopoietic tissue taken up by adipocytes (i.e., cellularity).

Fortunately, primarily because of the outstanding work of the University of Florida group, the state of affairs in marrow dosimetry has vastly improved over the past 2-3 y. This group has generated consistent anatomic models of the marrow on a macroscopic and cellular scale and has used these to perform 3-dimensional cell-level electron transport for several trabecular bone regions $(20,21)$. These models have been used to generate absorbed fractions that account for energy loss to cortical bone and also for marrow cellularity (22-24).

The paper in this issue of The Journal of Nuclear Medicine by Watchman et al. (25) adds to this group's already impressive contributions to this area. The article provides the basic data and describes a formalism for taking a step toward cell-level dosimetry while remaining within the overall MIRD $\mathrm{S}$ value formalism. As a step toward adjusting the mean absorbed dose to trabecular active marrow so as to account for the possibly nonuniform distribution of target cells, the distance between the trabecular bone surface and hematopoietic stem and progenitor cells and also their distance from blood vessels was measured by digital image analysis of immunohistochemically stained human bone marrow slides. The analysis showed an inverse linear relationship between distance from the trabecular bone surface and hematopoietic stem and progenitor cell density. In other words, the relevant target cells for hematopoietic suppression are not uniformly distributed throughout the marrow cavity but rather are concentrated close to the trabecular bone surface. Likewise, blood vessels were found more frequently near trabecular bone surfaces but with an exponential, 
rather than a linear, drop with distance from the bone surface. Another interesting observation was that the distance between blood vessels and hematopoietic stem and progenitor cells is lognormally distributed, meaning, as the authors point out, that there is a shared spatial niche between these 2 components of the bone marrow. The authors go on to show how these data could be used to scale bone marrow $\mathrm{S}$ values (e.g., trabecular bone surface source irradiating trabecular active marrow) to weight the estimated absorbed dose according to the spatial distribution of the relevant target cell population.

The objective of dosimetry in targeted radionuclide therapy is to provide information that will help improve patient care. With this objective, estimated absorbed dose is useful to the extent that it relates to response. The move from mean absorbed dose over an organ volume to an absorbed dose that is weighted to account for the spatial distribution of the relevant target cells is an important step in this direction, but more is needed. The conceptual 2-component framework of radionuclide dosimetry, requiring the physics of calculating absorbed fractions and $S$ values and the biology of determining the number of radionuclide transformations, must evolve to a 3-component framework that would take the resulting physics quantity absorbed dose and incorporate a third conceptual component, the radiobiologic modeling required to translate absorbed dose to biologic effect (26).

The translation of model and methodologic improvements to the clinic will require additional patient data. The sophisticated tools will not be useful if the input data required to apply them cannot be obtained or can be obtainable only at substantial logistic and monetary cost. This is perhaps the biggest challenge in the development of advanced dosimetric methods. Because of the costs, clinical implementation cannot be justified without an evaluation of the benefits. Benefits cannot be assessed unless the methodologies are widely implemented. With advances in technology, a focus on innovative approaches to find simpler data collection methods, the use of preclinical and postmortem studies, and knowledge gained from developments in external radiotherapy, we will surely find our way out of this catch-22.

George Sgouros
Johns Hopkins University School of Medicine
Baltimore, Maryland

\section{REFERENCES}

1. Marinelli LD, Quimby EH, Hine GJ. Dosage determination with radioactive isotopes. 2. Practical considerations in therapy and protection. AJR. 1948;59:260-281.

2. Loevinger R, Berman M. A Revised Schema for Calculating the Absorbed Dose from Biologically Distributed Radionuclides. MIRD Pamphet No. 1 (revised). New York, NY: The Society of Nuclear Medicine; 1976.

3. Snyder WS, Ford MR, Warner GG, Watson SB. "S" Absorbed Dose per Unit Cumulated Activity for Selected Radionuclides and Organs. MIRD Pamphlet No. 11. New York, NY: Society of Nuclear Medicine; 1975.

4. Cristy M, Eckerman KF. Specific Absorbed Fractions of Energy at Various Ages for Internal Photon Sources. Oak Ridge, TN: Oak Ridge National Laboratory; 1987. ORNL/TM-8381.

5. Eckerman KF, Stabin MG. Electron absorbed fractions and dose conversion factors for marrow and bone by skeletal regions. Health Phys. 2000;78:199-214.

6. Stabin MG. MIRDOSE: personal computer software for internal dose assessment in nuclear medicine. J Nucl Med. 1996;37:538-546.

7. Carrasquillo JA, White JD, Paik $\mathrm{CH}$, et al. Similarities and differences in In-111- and Y-90labeled 1B4M-DTPA antiTac monoclonal antibody distribution. J Nucl Med. 1999;40:268-276.

8. Sgouros G. Bone marrow dosimetry for radioimmunotherapy: theoretical considerations. $\mathrm{J} \mathrm{Nucl}$ Med. 1993;34:689-694.

9. Dewaraja YK, Ljungberg M, Majumdar A, Bose A, Koral KF. A parallel Monte Carlo code for planar and SPECT imaging: implementation, verification and applications in I-131 SPECT. Comput Methods Programs Biomed. 2002;67:115-124.

10. Sjogreen K, Ljungberg M, Strand SE. An activity quantification method based on registration of CT and whole-body scintillation camera images, with application to I-131. J Nucl Med. 2002;43:972-982.
11. He B, Frey EC. Comparison of conventional, model-based quantitative planar, and quantitative SPECT image processing methods for organ activity estimation using In-111 agents. Phys Med Biol. 2006;51:3967-3981.

12. Macey DJ, Grant EJ, Bayouth JE, et al. Improved conjugate view quantitation of I-131 by subtraction of scatter and septal penetration events with a triple energy window method. Med Phys. 1995;22: 1637-1643.

13. Siegel JA, Thomas SR, Stubbs JB, et al. MIRD pamphlet no. 16: techniques for quantitative radiopharmaceutical biodistribution data acquisition and analysis for use in human radiation dose estimates. J Nucl Med. 1999;40(suppl):37S-61S.

14. Stabin MG, Eckerman KF, Bolch WE, Bouchet LG, Patton PW. Evolution and status of bone and marrow dose models. Cancer Biother Radiopharm. 2002; 17:427-433.

15. Bouchet LG, Bolch WE, Howell RW, Rao DV. S values for radionuclides localized within the skeleton. J Nucl Med. 2000;41:189-212.

16. Spiers FW, Beddoe AH, Whitwell JR. Mean skeletal dose factors for beta-particle emitters in human bone. 1. Volume-seeking radionuclides. $\mathrm{Br}$ J Radiol. 1978;51:622-627.

17. Beddoe AH, Darley PJ, Spiers FW. Measurements of trabecular bone structure in man. Phys Med Biol. 1976;21:589-607.

18. Woodard HQ, Holodny E. A Summary of the data of Mechanik on the distribution of human bone marrow. Phys Med Biol. 1960;5:57-59.

19. Mechanik N. Studies of the weight of bone marrow in man [in German]. Zeitschrift fur die Gest Anatomy. 1926;79:58-99.

20. Jokisch DW, Bouchet LG, Patton PW, Rajon DA, Bolch WE. Beta-particle dosimetry of the trabecular skeleton using Monte Carlo transport within 3D digital images. Med Phys. 2001;28: 1505-1518.

21. Bouchet LG, Jokisch DW, Bolch WE. A threedimensional transport model for determining absorbed fractions of energy for electrons within trabecular bone. J Nucl Med. 1999;40:1947-1966.

22. Watchman CJ, Jokisch DW, Patton PW, Rajon DA, Sgouros G, Bolch WE. Absorbed fractions for alpha-particles in tissues of trabecular bone: considerations of marrow cellularity within the ICRP reference male. J Nucl Med. 2005;46:1171-1185.

23. Shah AP, Patton PW, Rajon DA, Bolch WE. Adipocyte spatial distributions in bone marrow: implications for skeletal dosimetry models. $\mathrm{J} \mathrm{Nucl}$ Med. 2003;44:774-783.

24. Bolch WE, Patton PW, Rajon DA, Shah AP, Jokisch DW, Inglis BA. Considerations of marrow cellularity in 3-dimensional dosimetric models of the trabecular skeleton. J Nucl Med. 2002;43:97-108.

25. Watchman CJ, Bourke VA, Lyon JR, et al. Spatial distribution of blood vessels and $\mathrm{CD} 34^{+}$hematopoietic stem and progenitor cells within the marrow cavities of human cancellous bone. $\mathrm{J} \mathrm{Nucl}$ Med. 2007;48:645-654.

26. Sgouros G. Dosimetry of internal emitters. J Nucl Med. 2005;46(suppl):18S-27S. 